\title{
Correlation of prefrontal cortical activation with changing vehicle speeds in actual driving: a vector-based functional near-infrared spectroscopy study
}

\author{
Kayoko Yoshino ${ }^{1}$, Noriyuki Oka ${ }^{1}$, Kouji Yamamoto ${ }^{2}$, Hideki Takahashi ${ }^{3}$ and Toshinori Kato ${ }^{1 *}$ \\ ${ }^{1}$ Department of Brain Environmental Research, KatoBrain Co., Ltd., Tokyo, Japan \\ ${ }^{2}$ Department of Environment/Engineering, Tokyo Branch, Central Nippon Expressway Co., Ltd., Tokyo, Japan \\ ${ }^{3}$ Department of Environment/Engineering, Central Nippon Expressway Co., Ltd., Nagoya, Japan
}

\section{Edited by: \\ Nobuo Masataka, Kyoto University, Japan \\ Reviewed by: \\ Yukiori Goto, Kyoto University, Japan Hirokazu Doi, Nagasaki University, Japan \\ ${ }^{*}$ Correspondence: \\ Toshinori Kato, Department of Brain Environmental Research, KatoBrain Co., Ltd., 13-15-104, Shirokanedai 3, Minato-ku, Tokyo, 108-0071, Japan e-mail: kato@katobrain.com}

Traffic accidents occur more frequently during deceleration than during acceleration. However, little is known about the relationship between brain activation and vehicle acceleration because it has been difficult to measure the brain activation of drivers while they drive. In this study, we measured brain activation during actual driving using vector-based functional near-infrared spectroscopy. Subjects decelerated from 100 to $50 \mathrm{~km} / \mathrm{h}$ (speed reduction task) and accelerated from 50 to $100 \mathrm{~km} / \mathrm{h}$ (speed increase task) while driving on an expressway, in the daytime and at night. We examined correlations between average vehicle acceleration in each task and five hemodynamic indices: changes in oxygenated hemoglobin $(\Delta \mathrm{oxy} H \mathrm{Hb})$, deoxygenated hemoglobin ( $\Delta$ deoxy $\mathrm{Hb}$ ), cerebral blood volume ( $\triangle \mathrm{CBV}$ ), and cerebral oxygen exchange ( $\triangle \mathrm{COE})$; and the phase angle $k$ (degrees) derived from the other hemoglobin $(\mathrm{Hb})$ indices. $\triangle \mathrm{oxyHb}$ and $\triangle \mathrm{CBV}$ reflect changes in cerebral blood flow, whereas $\Delta$ deoxy $\mathrm{Hb}, \Delta \mathrm{COE}$, and $k$ are related to variations in cerebral oxygen metabolism. Most of the resulting correlations with specific brain sites, for all the indices, appeared during deceleration rather than during acceleration. Faster deceleration resulted in greater increases in $\Delta$ deoxy $\mathrm{Hb}, \Delta \mathrm{COE}$, and $k$ in the prefrontal cortex $(r<-0.5, p<0.01)$, in particular, in the frontal eye field, and at night, it also resulted in greater decreases in $\triangle \mathrm{OxyHb}$ and $\triangle \mathrm{CBV}$ in the prefrontal cortex and in the parietal lobe $(r>0.4, p<0.01)$, suggesting oxygen metabolism associated with transient ischemic changes. Our results suggest that vehicle deceleration requires more brain activation, focused in the prefrontal cortex, than does acceleration. From the standpoint of the indices used, we found that simultaneous analysis of multiple hemodynamic indices was able to detect not only the blood flow components of hemodynamic responses, but also more localized frontal lobe activation involving oxygen metabolism.

Keywords: actual driving, supplementary eye field, outdoor brain activation, acceleration, deceleration, interregional correlation, phase angle, vehicle acceleration

\section{INTRODUCTION}

In recent years, neuroscience research related to vehicle driving has become popular. Brain function during driving encompasses responses to external stimuli such as visual stimulation and vehicle acceleration, in addition to internal processing involved in functions such as motor control and decision making. Functional neuroimaging during driving examines multiple intricate factors such as perception, cognition, thinking, and motor functions. It is important for road safety measures in the Intelligent Transport Systems (ITS) field to separate these factors and identify the regions of interest in the cerebral cortex for each of them.

Kato et al. (2013) and Yoshino et al. (2013) demonstrated that areas of the brain that are activated during acceleration were significantly different from those activated during deceleration in actual road experiments. That study suggested that brain activation during deceleration is higher than activation during acceleration; and this led us to investigate in more detail the relationship between brain activity and vehicle acceleration.
The most common cause of traffic accidents between vehicles in Japan is the rear-end collision (approximately $38.5 \%$ ) (Institute for Traffic Accident Research and Data Analysis, 2011). Rear-end collisions are caused by deceleration that occurs too late. It is possible that this is related to differences in brain function between acceleration and deceleration. However, no studies have yet examined brain responses during vehicle acceleration and deceleration in actual road driving.

Since there has been very little investigation of brain activity during actual road driving, the kind of responses that can be expected is still unclear. We thus decided to use fNIRS vector-based analysis, which is capable of explaining all the possible variations in the ratios of concentration changes in oxy $\mathrm{Hb}$ and deoxyHb (Kato, 2006, 2007). This is a method that displays compositely the hemodynamic response dues to changes in both oxyHb and deoxyHb on the same vector coordinate plane. Oxygenation and blood volume changes reflecting neural activity cannot be properly evaluated using the conventional analysis of 
either oxyHb or deoxyHb alone. In vector-based analysis, a cerebral blood volume (CBV) axis and a cerebral oxygen exchange (COE) axis are generated from an oxyHb and deoxyHb orthogonal coordinate plane, and the phase of vectors on this vector plane are evaluated (Yoshino and Kato, 2012; Sano et al., 2013), increasing the possible indices of brain activity. We thought it would be possible to use this method to evaluate brain activity during actual expressway driving from various perspectives.

We therefore investigated the relationship between brain regions and hemodynamic indices that increased or decreased in relation to calculated vehicle acceleration $\left(\mathrm{m} / \mathrm{s}^{2}\right)$ during tasks, using vector-based functional near-infrared spectroscopy (fNIRS) in a vehicle in an actual road experiment. This study aimed to extract brain responses and indices that are related to vehicle acceleration and deceleration by recording changes in cortical hemodynamic responses during driving by normal adults both during daytime and at night.

\section{METHODS \\ SUBJECTS}

Right-handed twelve healthy adults participated in this study (eight males and four females; average age, $33.3 \pm 4.5$ years). The subjects had operational experience on expressways and ordinary roads on a daily basis. The subjects had no history of mental or central nervous illnesses, and they took no medications on the day of the experiment. The subjects were comfortable in the experimental situation because they had previously participated in other actual expressway driving research (Yoshino et al., 2013) while wearing the fNIRS system probes. Written consent was obtained from the participants before enrollment in the study, and the protocol was approved in advance by the ethics committee at KatoBrain Co., Ltd. The subjects' average length of driving history was $11.8 \pm 5.8$ years. Their frequency of driving was $6.1 \pm 1.6$ times / week, and their frequency of expressway driving was $4.5 \pm 6.5$ times / month. Only two subjects had experienced an accident (neither accident involved another vehicle or any personal injuries), and the average number of accidents was $0.2 \pm 0.4$. The average number of traffic violations was $1.3 \pm 1.3$ times, mostly for speeding. Since recruitment of the subjects was based on the conditions of age, right-handedness, and frequency of driving on a daily basis, the subjects' genders, their driving histories, and their histories of violations and accidents were completely random.

\section{EXPERIMENTAL FIELD AND TEST VEHICLE}

The experiment was performed in the Okitsu district, Shizuoka Prefecture, Japan, on a section of the Shin Tomei Expressway immediately before it entered service (Yamamoto et al., 2012; Kato et al., 2013). Installation of signage, lighting and so on had already been completed, and so there were no problems with the safety of vehicle travel. For further safety, no vehicles were present other than the test vehicle on the experimental course. Guard personnel were located at each point on the experimental course, and they could immediately contact the test vehicle with a transceiver in any unexpected contingencies.

The experimental course was $2875 \mathrm{~m}$ long. It was almost straight, but included a gentle left and right curve $(R=5000$, both). The slope of the course was almost flat, but there were uphill and downhill gradients of $2.0 \%$. The test vehicle traveled in the left lane ( $3.75 \mathrm{~m}$ in width) in accordance with Japanese traffic regulations for lane use in two lanes. There was no artificial lighting provided on the experimental course, either in the daytime or at night.

An ordinary van, Hiace, which is made by the Toyota Motor Corporation (Japan) and is super-long with a high-roof specification, was used in this experiment. The vehicle was a twowheel-drive, gasoline-powered vehicle with a four-speed automatic transmission. A global positioning system receiver and a vehicle speed pulse counter were attached to the test vehicle, to record information on vehicle position, speed, and acceleration. Power was supplied to the fNIRS equipment by connecting a $\mathrm{DC} / \mathrm{AC}$ inverter to the battery of the vehicle.

\section{EXPERIMENTAL PROCEDURE}

The tasks included a speed reduction task of deceleration from 100 to $50 \mathrm{~km} / \mathrm{h}$ and a speed increase task of acceleration from 50 to $100 \mathrm{~km} / \mathrm{h}$. The speed increase task was performed immediately after the speed reduction task (Figure 1). One trial consisted of these two driving tasks, and six trials each were performed in the daytime and at night. Start and stop positions were provided on the course, and the subjects performed the two tasks at their own pace, with no cues on the course. After receiving their instructions and before putting on the fNIRS probes, the subjects performed 1-3 practice drives for the daytime and nighttime trials. The day and night experiments were performed on different days and the order of the experiments (day or night) was randomized between the subjects. The average duration of the speed increase task (acceleration) was $16.8 \pm 3.3 \mathrm{~s}$, and the average duration of the speed reduction task (deceleration) was $21.1 \pm 8.0 \mathrm{~s}$. The average ratio of acceleration time to deceleration time for all the subjects was $0.90 \pm 0.30$.

\section{fNIRS MEASUREMENTS AND REGISTRATION}

A multichannel fNIRS system (FOIRE-3000, Shimadzu Corporation, Japan) was mounted in the vehicle and used to measure hemodynamic responses. The equipment irradiated three wavelengths of NIR light $(780,805$, and $830 \mathrm{~nm})$ to the cerebral cortex, and monitored changes in the hemoglobin $(\mathrm{Hb})$ concentrations. Sampling intervals for measuring changes in $\mathrm{Hb}$ concentration were set to $70 \mathrm{~ms}$.

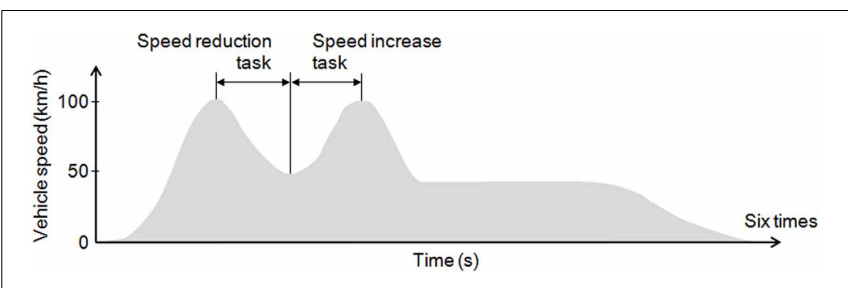

FIGURE 1 | Experimental tasks. Each subject performed 6 daytime trials and 6 nighttime trials on the experimental course. The tasks included a speed reduction task of deceleration from 100 to $50 \mathrm{~km} / \mathrm{h}$ and a speed increase task of acceleration from 50 to $100 \mathrm{~km} / \mathrm{h}$. 
The fNIRS device was tightly secured to the vehicle using two bars installed behind the driver's seat and a hook on the floor of the vehicle. The probe line was also attached to the bars behind the driver's seat. Probes were attached to the subject's head in a way that allowed for moderate changes in driving posture. To prevent noise due to sunlight, the front and rear of the device, and the subject's head were covered with black cloth after the probes were attached.

Measurement areas were located on both sides of the prefrontal cortex, and on the motor cortex and the parietal cortex; the occipital lobe was excluded for the safety of the subjects (Figure 2A). Forty-eight channels were set up using 16 irradiation and 16 detection probes. The distance between irradiation and detection probes was $3 \mathrm{~cm}$.

Confirmation of the position of each measurement point was done by magnetic resonance imaging (MRI), using a 3-Tesla 3D-T2-weighted MRI system (Philips Co., Achieva 3.0 Quasar Dual 3.0T-MRI), in which the subject probe attachments were fitted with registration markers. The sampling conditions used the spin-echo method with an echo time of $247 \mathrm{~ms}$, a repetition time of $2700 \mathrm{~ms}$, image size of $250 \times$ 250 pixels, a slice thickness of $1.0 \mathrm{~mm}$ in the sagittal direction, and an interslice gap of $0 \mathrm{~mm}$. As Figure 2B shows, the positions of the probes were confirmed for all of the measured areas based on the locations of the registration markers. Figure 2C shows the Brodmann areas (BA) having the highest correspondence with each channel, from the MRIs of all the subjects.

\section{ANALYSIS}

\section{The vector approach}

In the blood vessels, changes in oxygenation and blood volume occur in response to neural activity, and changes in the concentrations of both oxyHb and deoxyHb are involved in this response. To detect changes in both oxygenation and blood volume, the following vector analysis method was used.
$\mathrm{OxyHb}$ and deoxyHb have different chemical properties (paramagnetic or diamagnetic) that are due to differences in the bonding of oxygen molecules (Pauling and Coryell, 1936). Taking this into consideration, an orthogonal vector coordinate plane is set up, defined by oxyHb $(\Delta O)$ and deoxyHb $(\Delta D)$ axes (Kato, $2006,2007)$. As Figure 3 shows, rotating this $\Delta O / \Delta D$ vector plane 45 degrees counterclockwise results in an orthogonal vector coordinate plane comprising a $(\Delta O+\Delta D)$ axis and a $(\Delta D-\Delta O)$ axis. A vector $(\Delta O+\Delta D)$ can be defined as a cerebral blood volume $(\Delta \mathrm{CBV})$ vector, and a vector $(\Delta D-\Delta O)$ can be defined as a cerebral oxygen exchange vector $(\triangle \mathrm{COE})$. A positive value for $\triangle \mathrm{CBV}$ indicates locally increasing $\triangle \mathrm{CBV}$ and a negative value

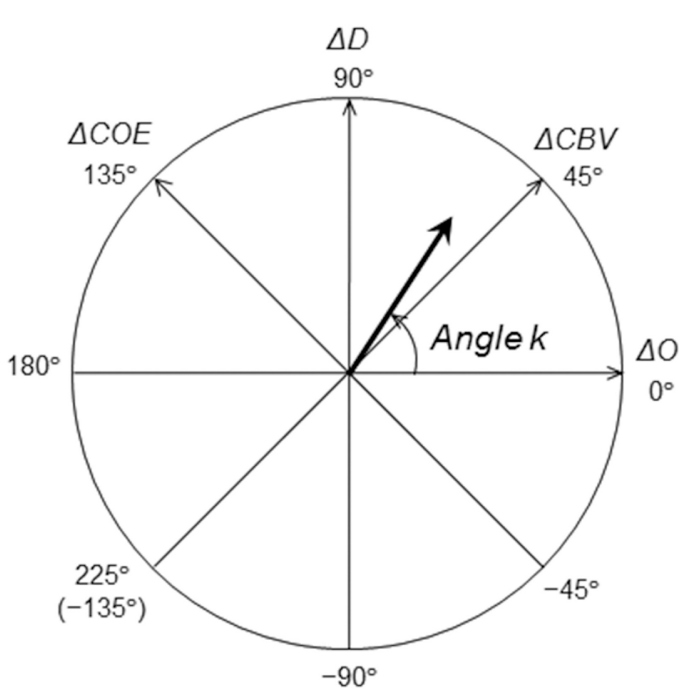

FIGURE 3 | Definition of the vector coordinates. Polar coordinate plane for the analysis of cerebral oxygenation and blood volume. The relationship between cerebral oxygen exchange $(\triangle \mathrm{COE})$ and cerebral blood volume $(\triangle \mathrm{CBV})$ can be detected by vector trajectories.
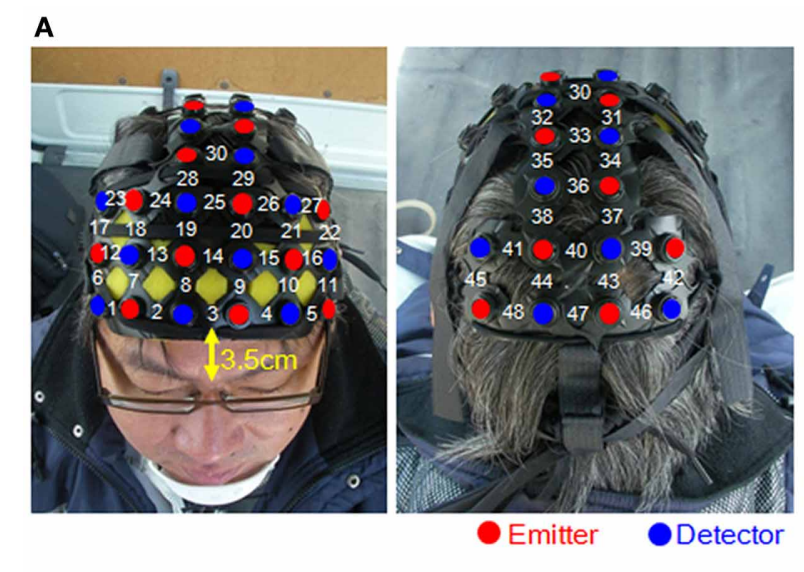

FIGURE 2 | Measurement sites and the locations of the probes.

(A) Head probe attachments and channel numbers. (B) Positioning of the probes was confirmed using MRI. (C) Dashed curves represent the
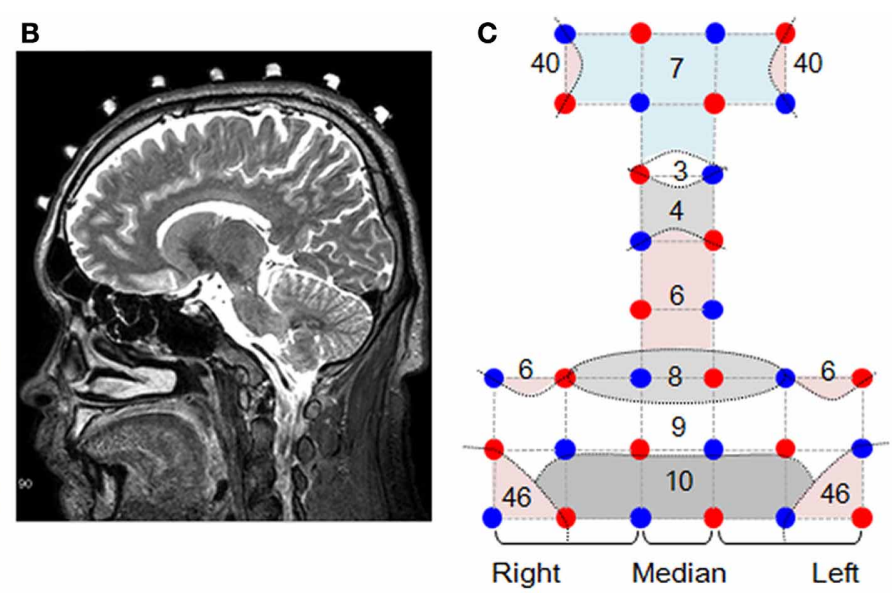

schematic boundaries of the Brodmann areas that were confirmed by MRI. Straight lines connecting the emitters and detectors indicate channel positions. 
for $\triangle \mathrm{CBV}$ indicates decreasing $\triangle \mathrm{CBV}$. A positive value of $\triangle \mathrm{COE}$ indicates hypoxic change from $\triangle \mathrm{COE}=0$, and a negative value of $\triangle \mathrm{COE}$ indicates hyperoxic change. The relationship among the four axes of $\Delta O, \Delta D, \triangle \mathrm{CBV}$, and $\Delta \mathrm{COE}$ is described by the following square matrix:

$$
\begin{aligned}
\left(\begin{array}{c}
\Delta O+\Delta D \\
-\Delta O+\Delta D
\end{array}\right) & =\left(\begin{array}{cc}
1 & 1 \\
-1 & 1
\end{array}\right)\left(\begin{array}{l}
\Delta O \\
\Delta D
\end{array}\right)=\left(\begin{array}{l}
\Delta \mathrm{CBV} \\
\Delta \mathrm{COE}
\end{array}\right) \\
\left(\begin{array}{c}
\Delta O \\
\Delta D
\end{array}\right) & =\frac{1}{2}\left(\begin{array}{cc}
1 & -1 \\
1 & 1
\end{array}\right)\left(\begin{array}{l}
\Delta \mathrm{CBV} \\
\Delta \mathrm{COE}
\end{array}\right)
\end{aligned}
$$

These can be expanded to obtain $\triangle \mathrm{CBV}$ and $\triangle \mathrm{COE}$ as follows.

$$
\begin{aligned}
& \Delta \mathrm{CBV}=\frac{(\Delta D+\Delta O)}{\sqrt{2}} \\
& \Delta \mathrm{COE}=\frac{(\Delta D-\Delta O)}{\sqrt{2}}
\end{aligned}
$$

The phases (octants) on the vector plane described above provide a quantitatively defined representation of the degree of oxygen exchange; the phase of a vector is defined by the angle $k$ (degrees), which is a ratio of $\Delta \mathrm{D}$ to $\Delta \mathrm{O}$, and it reflects the strength of oxygen metabolism. $k$ is the angle between a vector and the positive $\Delta \mathrm{O}$ axis, and it is determined as follows:

$$
\begin{aligned}
k & =\operatorname{Arctan}\left(\frac{\Delta D}{\Delta O}\right) \\
& =\operatorname{Arctan}\left(\frac{\Delta \mathrm{COE}}{\Delta \mathrm{CBV}}\right)+45^{\circ} \quad\left(-135^{\circ} \leqq k \leqq 225^{\circ}\right)
\end{aligned}
$$

$k=0^{\circ}$ is on the positive $\Delta O$ axis, and coincides with the oxygen density of arterial blood. An increase in $k$ is defined within the range of increase in $\Delta D$ or $\Delta \mathrm{COE}\left(0^{\circ} \leqq k \leqq 235^{\circ}\right)$, and a decrease in $k$ is defined within the range of decrease in $\Delta D$ and $\triangle \mathrm{COE}\left(-135^{\circ} \leqq k \leqq 0^{\circ}\right)$. An increase or decrease in $k$ thus indicates a change in oxygen demand.

\section{Data processing and statistics}

The $\Delta D$ and $\Delta O$ data were subjected to low-pass filtering at $0.1 \mathrm{~Hz}$ to remove any high frequency components. For each of these two indicators, the average changes per second among the tasks were determined for each channel. $\triangle \mathrm{COE}, \triangle \mathrm{CBV}$, and $k$ were calculated using these data. In this process, the actual changes for each task were calculated by setting the levels to zero at the beginning of each task.

Average vehicle acceleration $\left(\mathrm{m} / \mathrm{s}^{2}\right)$ during each task was determined by Equation (6). Positive values indicate average vehicle acceleration during speed increase tasks, and negative values indicate average vehicle acceleration during speed reduction tasks.

$$
\begin{aligned}
& \text { Average vehicle acceleration } \\
& \qquad=\text { (initial velocity - final velocity)/time }
\end{aligned}
$$

Analyses of correlations (Spearman's rank correlation coefficient) between average vehicle acceleration and each hemodynamic index were performed. Analyses of interregional correlations (Spearman's rank correlation coefficient) within each hemodynamic index were also performed. These tests were applied separately to each of the daytime and nighttime experiments. The data used for analysis was a total of 70 trials in the daytime and 70 trials in nighttime. Two trials each in the daytime and nighttime experiments were excluded because $\mathrm{Hb}$ monitoring was not successful. The significance level was set to $5 \%$. Correlation coefficients that were higher than \pm 0.4 were evaluated.

\section{RESULTS}

\section{AVERAGE VEHICLE ACCELERATION}

Table 1 shows average vehicle acceleration during the two tasks. Negative values indicate deceleration. There were no significant differences between daytime and nighttime for either of the tasks.

\section{CORRELATIONS IN THE VEHICLE SPEED REDUCTION TASK}

Figure 4 shows mapping images of the correlations between average vehicle acceleration and each of the hemodynamic indices in the speed reduction task. The results divide into significant negative and positive correlations according to the indices used. Significant negative correlations were observed for $\triangle \mathrm{COE}, \Delta D$, and $k(p<0.01)$. Significant positive correlations were observed for $\Delta \mathrm{CBV}$ and $\Delta O(p<0.01)$.

\section{Negative correlations in the vehicle speed reduction task}

Table 2 shows correlations of -0.4 or lower in the speed reduction task. The greater the deceleration, the more $\Delta \mathrm{COE}, \Delta D$, and $k$ increased. These correlations were observed only in the frontal lobe (BA10, BA9, BA8 and BA6), and none were observed in the parietal lobe.

In the speed reduction task, high negative correlations were observed between $\triangle \mathrm{COE}$ and vehicle acceleration in the medial BA8 (daytime: $r=-0.545, p<0.001$; nighttime: $r=-0.670$, $p<0.001)$. In the nighttime results, in addition to the negative correlations observed in the medial BA8, negative correlations increased in the peripheral regions of the left BA8 (Ch. 26), the left BA9 (Ch. 20), and the left BA6 (Ch. 29) $(-0.622<r<-0.613$, $p<0.001)$. These correlations during the nighttime tasks were

\begin{tabular}{|c|c|c|c|c|}
\hline \multirow[t]{2}{*}{ Tasks } & \multicolumn{2}{|c|}{ Vehicle acceleration $\left(\mathrm{m} / \mathrm{s}^{2}\right)$} & \multirow[t]{2}{*}{$\boldsymbol{t}$} & \multirow[t]{2}{*}{$\boldsymbol{p}$} \\
\hline & Day time & Night time & & \\
\hline Speed reduction task & $-0.75 \pm 0.27$ & $-0.76 \pm 0.30$ & 0.182 & 0.856 (n.s.) \\
\hline Speed increase task & $0.86 \pm 0.18$ & $0.85 \pm 0.16$ & 0.291 & 0.772 (n.s.) \\
\hline
\end{tabular}
also observed with $\Delta D$ and $k(\Delta D:-0.661<r<-0.543, p<$ $0.001 ; k:-0.596<r<-0.431, p<0.001)$. Negative correlations with vehicle acceleration were also observed with $\triangle \mathrm{COE}$ and $k$ at night in the medial BA10 (Ch. 3) and in the left BA10 (Chs. 4 and 9) ( $\triangle$ COE: $-0.698<r<-0.604, p<0.001 ; k$ : $-0.614<r<-0.539, p<0.001)$.

\section{Table 1 | Average vehicle acceleration.}

n.s., not significant. 
A

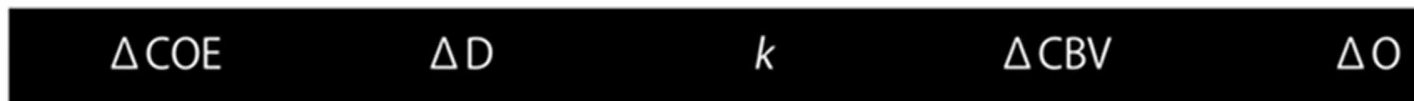

\section{Day}
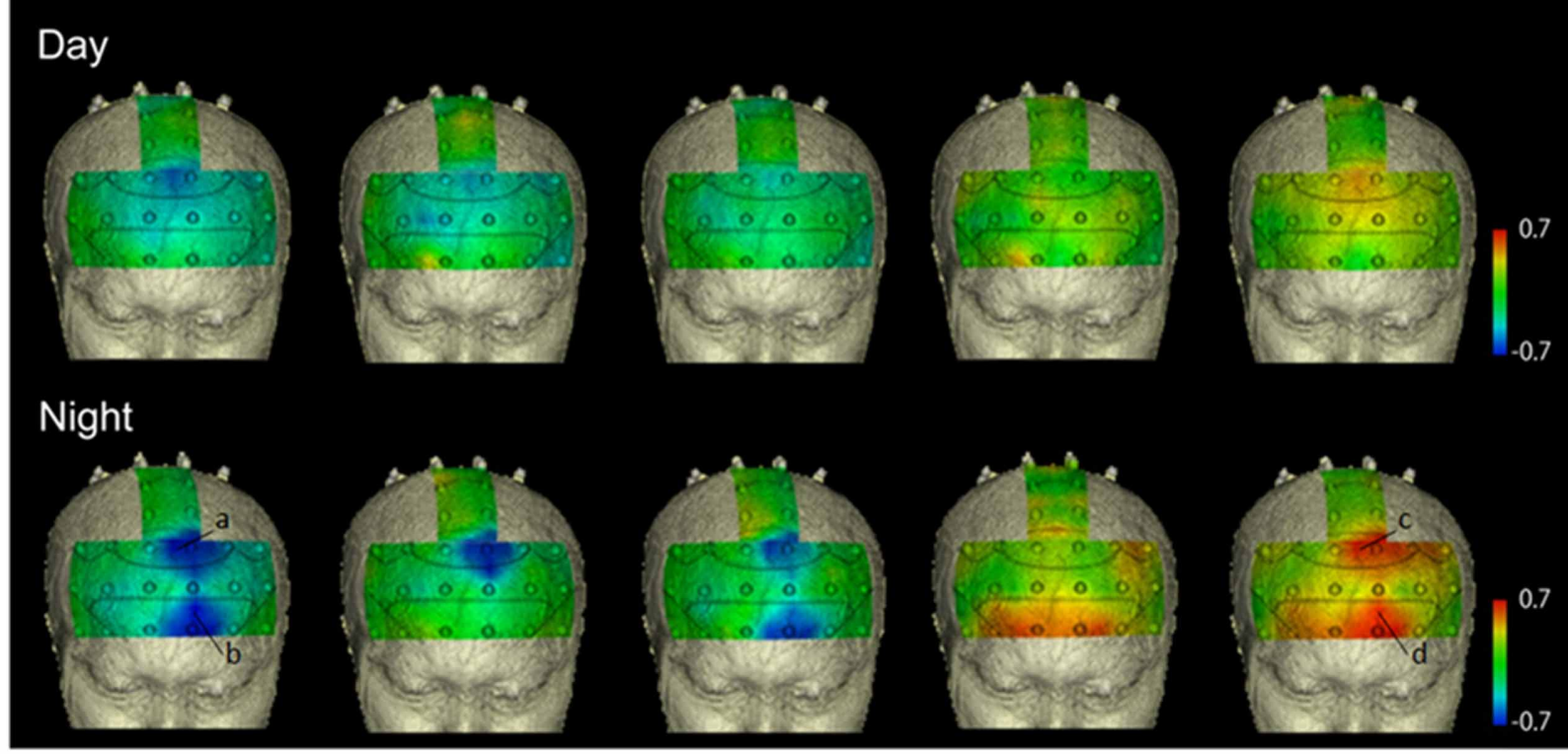

B

a: $\triangle \operatorname{COE}($ Ch. 25)

b: $\triangle \operatorname{COE}(\mathrm{Ch} .9)$

c: $\Delta \mathrm{O}$ (Ch. 25)

d: $\Delta \mathrm{O}$ (Ch. 9)
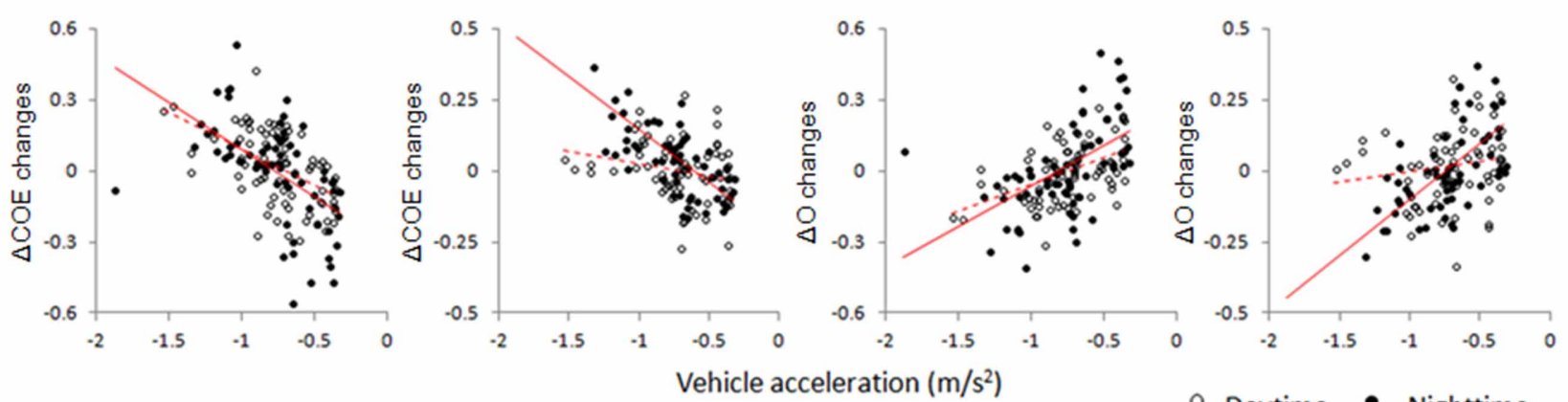

… Daytime $\bullet$ Nighttime

FIGURE 4 | Correlations in the speed reduction task between vehicle acceleration and each of the brain activation indices. (A) Correlation maps showing correlations between vehicle acceleration and each of the hemodynamic indices in the speed reduction task. (B) Correlation diagrams from the medial BA8 (Ch. 25) and the left BA10 (Ch. 9) showed marked correlation with vehicle acceleration. Vehicle acceleration correlated negatively with $\triangle \mathrm{COE}$ and positively with $\Delta \mathrm{O}$. Correlation coefficients were higher at night than in the daytime.

\section{Positive correlations in the speed reduction task}

Table 3 shows correlations 0.4 or more in the speed reduction task. The greater the deceleration, the more $\triangle \mathrm{CBV}$ and $\triangle O$ decreased. Activation occurred during the daytime tasks mainly on the periphery of the medial BA8 $(r=0.464, p<0.001)$; and during the nighttime tasks, in the medial and the left BA8 and the area surrounding them $(0.436<r<0.654, p<0.001)$ and in both the right and left BA10 $(0.486<r<0.674, p<0.001)$. Positive correlations were also observed for $\triangle \mathrm{CBV}$ in both the right and the left BA10 at night $(0.422<r<0.601, p<0.001)$.

\section{CORRELATIONS IN THE SPEED INCREASE TASK}

Figure 5 shows mapping images of the correlations between average vehicle acceleration and each of the hemodynamic indices in the speed increase task. Table 4 shows the correlations of 0.4 or more in this task.
This task did not divide into positive and negative correlations depending on the index used. There were no correlation coefficients greater than \pm 0.5 in the speed increase task, except for $\triangle \mathrm{CBV}$ in BA46. The only reproducible correlations found in either the daytime or nighttime tasks were in the left BA46. In the left BA46 (Ch. 5), $\triangle O$ demonstrated a negative correlation with vehicle acceleration in both the daytime and nighttime tasks (daytime: $r=-0.465, p<0.001$; nighttime: $r=-0.412, p<0.001)$. The more rapid the acceleration, the more $\Delta O$ decreased in the left BA46.

\section{INTERREGIONAL CORRELATIONS WITH THE SUPPLEMENTARY EYE FIELD}

Figure 6 shows mapping images of the correlations between responses in the supplementary eye field (medial BA8, where there was a high correlation with vehicle acceleration in both the 
Table 2 | Negative correlations in the speed reduction task between vehicle acceleration and the brain activation indices.

\begin{tabular}{|c|c|c|c|c|c|c|c|c|}
\hline & & \multirow[t]{2}{*}{ Ch. } & \multicolumn{2}{|c|}{$\Delta \mathrm{COE}$} & \multicolumn{2}{|c|}{$\Delta D$} & \multicolumn{2}{|r|}{$k$} \\
\hline & & & Day & Night & Day & Night & Day & Night \\
\hline \multirow[t]{3}{*}{ BA10 } & Med. & 3 & & $-0.604^{*}$ & & & & $-0.597^{*}$ \\
\hline & Left & 4 & & $-0.663^{*}$ & & & & $-0.614^{*}$ \\
\hline & & 9 & & $-0.698^{*}$ & & & & $-0.539 *$ \\
\hline
\end{tabular}

\begin{tabular}{|c|c|c|c|c|c|c|c|}
\hline \multirow[t]{2}{*}{ BA9 } & \multirow{2}{*}{$\begin{array}{l}\text { Right } \\
\text { Left }\end{array}$} & \multicolumn{2}{|l|}{13} & \multicolumn{3}{|c|}{$-0.493^{*}$} & \multirow[b]{2}{*}{$-0.462 *$} \\
\hline & & 20 & & $-0.613^{*}$ & & $-0.661^{*}$ & \\
\hline \multirow[t]{2}{*}{ BA8 } & Med. & 25 & $-0.545^{*}$ & $-0.670^{*}$ & $-0.457^{*}$ & $-0.603^{*}$ & $-0.596^{*}$ \\
\hline & Left & 26 & & $-0.613^{*}$ & & $-0.603^{*}$ & $-0.431 *$ \\
\hline BA6 & Left & 27 & $-0.413^{*}$ & & $-0.424^{*}$ & & \\
\hline
\end{tabular}

${ }^{*}$ Correlations of $r<-0.4$ were all significant at $p<0.01$.

daytime and nighttime tasks) and responses in the other areas, during the speed reduction task. Table 5 shows the number of channels with correlations of 0.4 or more with the medial BA8 $(r>0.4)$, by index.

Interregional correlations with $k$ were identified in 4-6 channels (daytime and nighttime tasks). For the other Hb indices, the number of correlations with the medial BA8 ranged from 11 to 23 channels. Particularly for $\triangle O$ and $\triangle \mathrm{CBV}$, localization was poor.

For $k$ in the daytime speed reduction task, positive correlations with the medial BA8 were identified in the left BA8 (Ch. 26; $r=0.415$ ), the left BA6 (Ch. 29; $r=0.657$ ), the medial BA10 (Ch. 3; $r=0.415$ ), and the left BA10 (Ch. 4; $r=0.446$ ) $(p<0.01)$. These correlations were higher at night $(0.477<r<$ $0.741)$. Correlations also occurred in the area surrounding the left BA10 (Ch. 4; $r=0.630$ ) and in BA9 (Ch. 20; $r=0.533$ ) $(p<0.01)$.

\section{DISCUSSION \\ RELATIONSHIP BETWEEN CHANGING VEHICLE SPEEDS AND BRAIN ACTIVATION}

Correlations between the hemodynamic indices and vehicle acceleration were found to be higher in the speed reduction task, and lower in the speed increase task. In the speed reduction task, there were negative correlations between vehicle acceleration and the indices $\triangle \mathrm{COE}$ and $k$ (that is, $\triangle \mathrm{COE}$ and $k$ showed greater increases during faster deceleration). Because $\triangle \mathrm{COE}$ and $k$ are indicators of change in oxygen metabolism, this suggests that oxygen metabolism increased during rapid deceleration of the vehicle.

Areas that typically exhibited greater increases in oxygen metabolism during vehicle speed reduction were BA8 and its surrounding area (including BA9 and BA6), which are involved in eye movement (Fukushima et al., 2000, 2002; Pierrot-Deseilligny et al., 2004). The field of view is narrower when the vehicle is driven at a high speed, while it spreads gradually, approaching a steady state when the vehicle speed decreases. BA8 controls sideto-side eye movements, and vergence eye movements responsible for depth perception (Gamlin and Yoon, 2000). The element of
Table 3 | Positive correlations in the speed reduction task between vehicle acceleration and each of the brain activation indices.

\begin{tabular}{|c|c|c|c|c|c|c|}
\hline & & \multirow[t]{2}{*}{ Ch. } & \multicolumn{2}{|c|}{$\Delta \mathrm{CBV}$} & \multicolumn{2}{|c|}{$\Delta O$} \\
\hline & & & Day & Night & Day & Night \\
\hline \multirow[t]{5}{*}{ BA10 } & Right & 2 & $0.434^{*}$ & $0.594^{*}$ & & $0.512 *$ \\
\hline & & 7 & & $0.489 *$ & & $0.486^{*}$ \\
\hline & & 8 & & $0.459 *$ & & \\
\hline & Left & 4 & & $0.601^{*}$ & & $0.674^{*}$ \\
\hline & & 9 & & $0.422^{*}$ & & $0.650 *$ \\
\hline \multirow[t]{2}{*}{ BA9 } & Left & 20 & & & & $0.498^{*}$ \\
\hline & & 21 & & $0.419^{*}$ & & \\
\hline BA46 & Right & 1 & & $0.476^{*}$ & & $0.436 *$ \\
\hline \multirow[t]{2}{*}{ BA8 } & Med. & 25 & & & $0.464^{*}$ & $0.654 *$ \\
\hline & Left & 26 & & & & $0.617^{*}$ \\
\hline \multirow[t]{3}{*}{ BA6 } & Med. & 30 & & $0.484^{*}$ & & \\
\hline & Left & 27 & & $0.489 *$ & & $0.528 *$ \\
\hline & & 29 & & & & $0.650^{*}$ \\
\hline BA3 & Med. & 36 & $0.424 *$ & & $0.442 *$ & \\
\hline \multirow[t]{2}{*}{ BA7 } & Med. & 40 & & $0.410^{*}$ & & \\
\hline & Left & 44 & & $0.406^{*}$ & & $0.414^{*}$ \\
\hline
\end{tabular}

${ }^{*}$ Correlations of $r<-0.4$ were all significant at $p<0.01$.

vergence eye movements is particularly important in fast vehicle traveling, but a more balanced ratio between the two types of eye movements would be required in slow vehicle traveling. This suggests that a spread in the direction of eye movement control occurs along with the spread in the field of view that occurs during rapid deceleration, and the activation of BA8 possibly increases at that time. In contrast, a possible reason for the low correlation between vehicle acceleration (positive acceleration) and the activation in BA8 during the vehicle speed increase task is that the acceleration of the vehicle results in a narrowing of the field of view, and the direction of the control direction easily becomes fixed in one direction.

Changes in vision enter the driver's brain as movement of the optic flow. It can be hypothesized that the increase in oxygen metabolism in BA8 results from the optic flow that is derived from changes in vehicle speed. The relationship between BA8 and the optic flow may be a key point in future research, as one of the regions of interest in the neuroimaging research on the driver's brain.

During nighttime driving, there were more correlations in the area surrounding BA8 than during daytime driving, and there were also more correlations between vehicle deceleration and oxygen metabolism in BA10, which is involved in executive function. During rapid deceleration at night, enhanced attention, or increased awareness of one's visual field and adjustments to one's eye movements may be more necessary than in the daytime. BA10 is known to be activated more in dual tasks (Baddeley and Della Sala, 1996). Vehicle deceleration at night requires control of the vehicle speed on a dark road without artificial lighting, and 


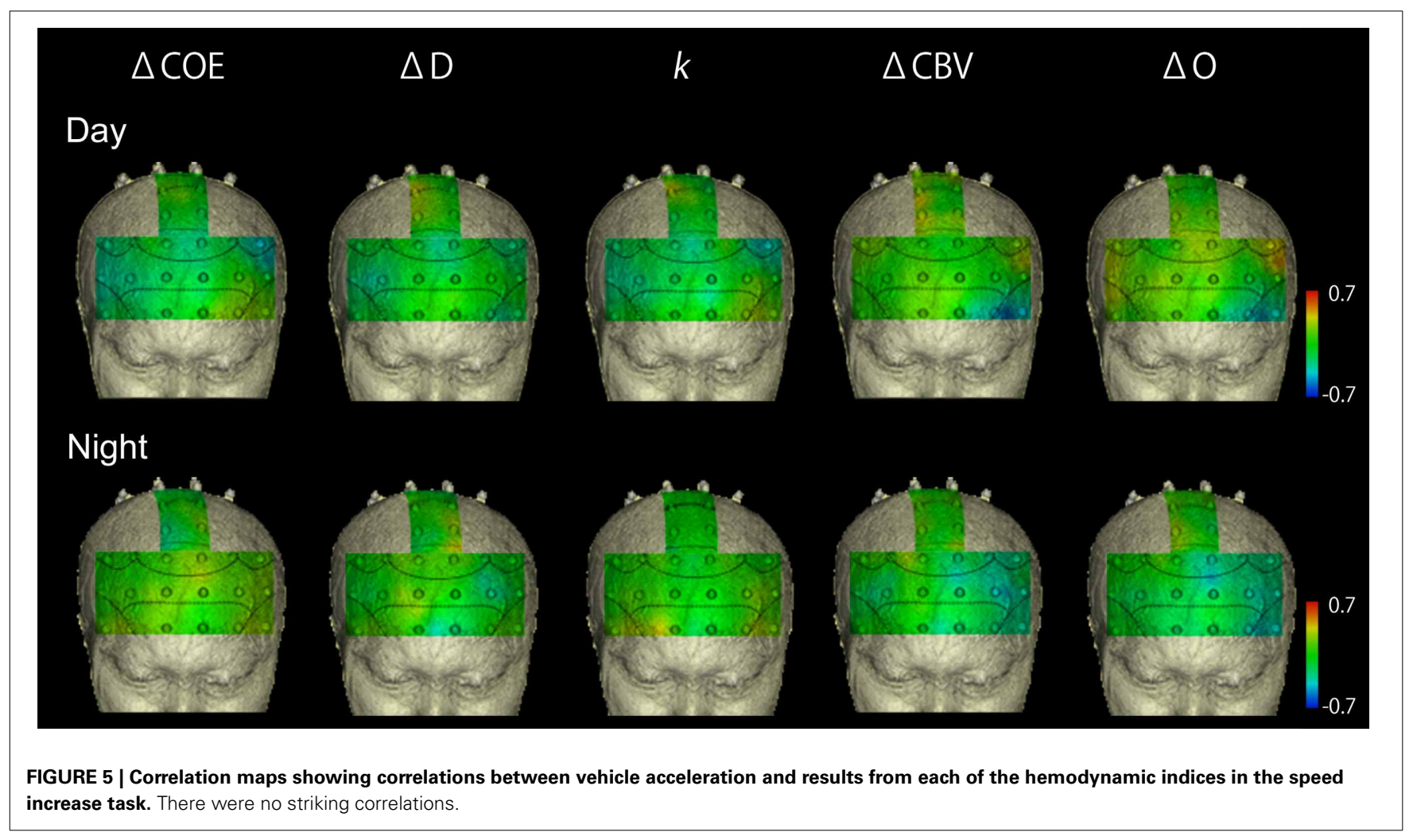

Table 4 | Correlations in the speed increase task between vehicle acceleration and each of the brain activation indices $(p<0.01)$.

\begin{tabular}{|c|c|c|c|c|c|c|c|c|}
\hline & & \multirow[t]{2}{*}{ Ch. } & \multicolumn{2}{|c|}{$\Delta \mathrm{COE}$} & \multicolumn{2}{|c|}{$\Delta \mathrm{CBV}$} & \multicolumn{2}{|c|}{$\Delta O$} \\
\hline & & & Day & Night & Day & Night & Day & Night \\
\hline \multirow[t]{3}{*}{ BA9 } & Left & 16 & & & & $-0.445^{*}$ & & \\
\hline & & 20 & & & & & & $-0.443^{*}$ \\
\hline & & 22 & $-0.416^{*}$ & & & & $0.454^{*}$ & \\
\hline BA46 & Left & 5 & & & $-0.544^{*}$ & & $-0.465^{*}$ & $-0.412^{*}$ \\
\hline BA7 & Left & 44 & & & & $0.436^{*}$ & & $0.461 *$ \\
\hline
\end{tabular}

${ }^{*}$ Correlations of $r<-0.4$ were all significant at $p<0.01$.

in addition, the useful field of view at night is narrower than in the daytime. It is thus likely that increased involvement of BA10 in deceleration at night occurs because the driver's attention must be allocated to a greater extent to both visual and motor control. Additional differences between daytime and nighttime driving may also exist in sites that were not included in our measurements.

\section{INTERREGIONAL CONNECTIVITY AND THE DIFFERENT BRAIN ACTIVATION INDICES}

Interregional correlations between brain activation in BA8 and BA10 in both the daytime and at night were shown by the angle $k$. Correlations with vehicle acceleration, however, were observed in BA8 only during the day and in both BA8 and 10 at night. This suggests the possibility of a functional connectivity between BA8 and 10 regardless of the presence or absence of a correlation with vehicle acceleration. Anatomically, in addition to BA8, the frontal eye field also includes BA6 and BA9 (Goldberg et al., 1991). This could explain why correlations were higher with the areas surrounding BA8: BA6 (Ch. 29) and BA9 (Ch. 20). BA10 has been considered to be a separate area from BA8, and the detection of functional connectivity between these areas is thus a new finding. Further investigation of this point will be necessary.

The present study demonstrated that the identification of relationships between local brain activation differs according to the hemodynamic index used. Localized interregional relationships were shown by the angle $k$ (degrees), which reflects variation in oxygen metabolism. The relationships shown by $\triangle \mathrm{CBV}$ and $\triangle O$, however, covered most of the prefrontal cortex. Hemodynamic responses in the blood vessels contain an oxygen metabolic component and a blood flow component. Differences in $\triangle \mathrm{CBV}$ reflect the blood flow factor, and differences in $\triangle \mathrm{COE}$ reflects the oxygen metabolism factor. In other words, a hemodynamic index must include both indicators that reflect oxygen metabolic relationships and indicators that reflect vascular relationships. These factors are determined by the ratio of the variations in deoxyHb and oxyHb, and this is the ratio $k$ on which the concept of phase is based. In the results of this study, the use of $\triangle \mathrm{CBV}$ and $\triangle O$ reflected vascular relationships, and $\triangle \mathrm{COE}$ and $k$ were likely reflected oxygen metabolic relationships.

To date, fNIRS studies have frequently been conducted using time series correlations of oxyHb to detect functional connectivity (Homae et al., 2010). Increases in oxyHb, however, are related to the inflow of arterial blood, and thus do not necessarily reflect 


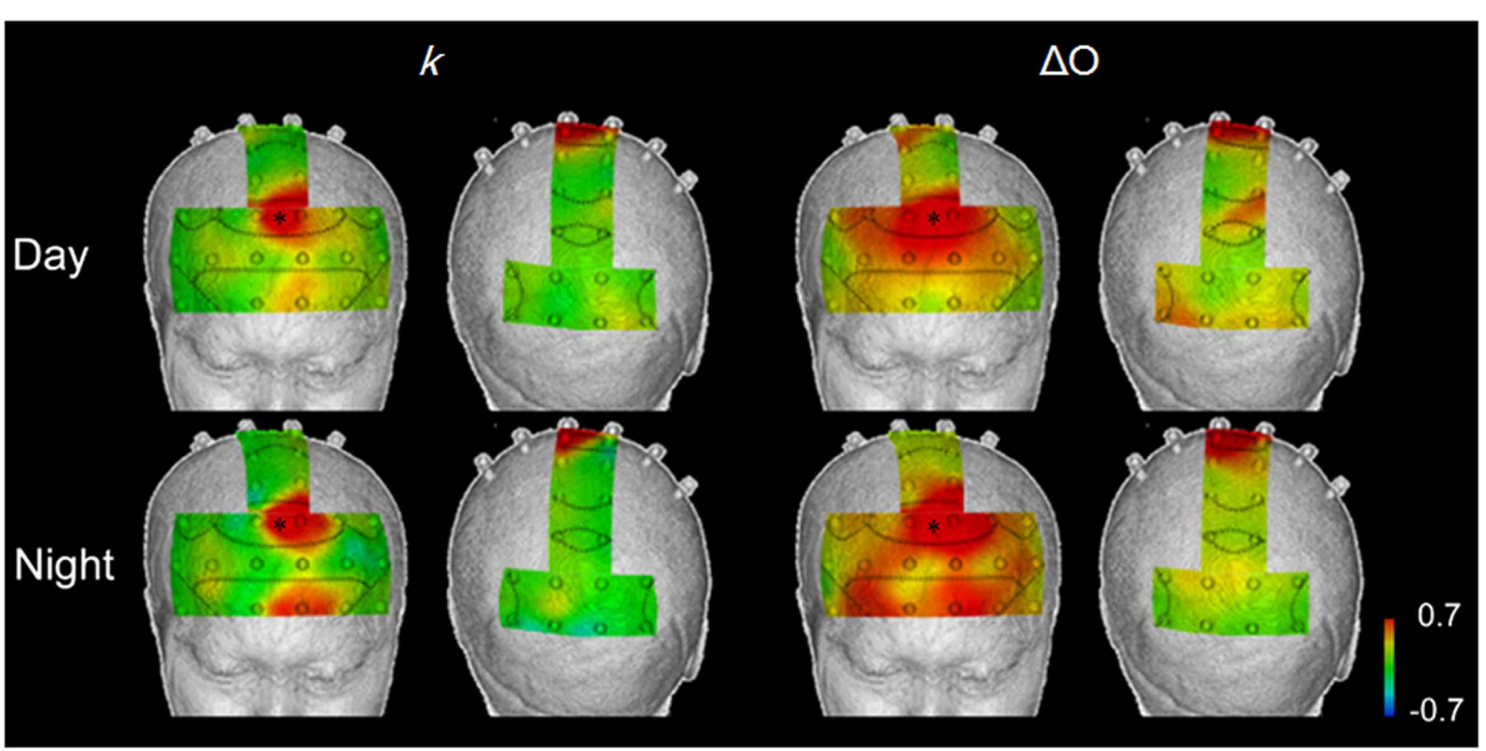

FIGURE 6 | Correlation maps showing interregional correlations between responses in the supplementary eye field $\left({ }^{*}\right)$, where there were high correlations with vehicle acceleration during in both daytime and nighttime trials, and the responses in other areas, in the speed reduction task, using $\boldsymbol{k}$ and $\boldsymbol{\Delta} \boldsymbol{O}$. $k$ showed localized areas of correlation, while $\Delta O$ showed broad correlations in the prefrontal cortex.
Table 5 | Number of channels showing positive correlations with the medial BA8 $(r>0.4)$.

\begin{tabular}{|c|c|c|c|c|c|c|c|c|c|c|}
\hline & & $k$ & & $\Delta D$ & & COE & & CBV & & 0 \\
\hline & Day & Night & Day & Night & Day & Night & Day & Night & Day & Night \\
\hline BA10 & 2 & 3 & & & 3 & 6 & 2 & 5 & 5 & 6 \\
\hline BA9 & & 1 & 5 & 1 & 6 & 6 & 9 & 6 & 8 & 6 \\
\hline BA46 & & & 1 & & 1 & 1 & 1 & 3 & & 2 \\
\hline BA8 & 1 & 1 & 1 & 1 & 2 & 1 & 2 & & 2 & 2 \\
\hline BA6 & 1 & 1 & 4 & 1 & 2 & 1 & 6 & 3 & 3 & 4 \\
\hline BA4 & & & & & & & & & 1 & \\
\hline BA3 & & & & & 1 & & & & 1 & \\
\hline BA7 & & & & & & & 2 & 1 & 2 & \\
\hline BA40 & & & & & & & & 1 & 1 & \\
\hline Total & 4 & 6 & 11 & 3 & 15 & 15 & 22 & 19 & 23 & 20 \\
\hline
\end{tabular}

increases in neural activity (Kato, 2004). Consequently, because these correlations based on oxyHb cannot distinguish actual activation from vascular networks in the scalp or the brain surface, the possibility that vascular relationships have been overestimated as functional connectivity cannot be denied. Skin blood flow are likely to be incorporated into measurements of oxyHb (Takahashi et al., 2011; Kirilina et al., 2012), and thus the use of phase, based on the angle $k$, has been proposed as a solution (Sano et al., 2013). In future research, the presence of differences in functional brain imaging based on these indices will need to be addressed as a technical and physiological issue.

Furthermore, in the present study we examined correlations using variation per second within the tasks, and this is another issue that it will be necessary to reconsider, with a view toward time series correlations in the future.

\section{THE SIGNIFICANCE OF NEUROSCIENCE FINDINGS IN ITS}

Since research on the actual expressway driving is still in its infancy, the brain scientific findings are obviously still insufficient in the field of ITS as it relates to expressway construction and management. In particular, there is little basic knowledge about influences on the brain from the behavior of the vehicle in actual highway driving. There has been a simulation experiment on expressway driving using fMRI (Graydon et al., 2004), but it does not include the actual physical driving operations, and the field of view is different from that in actual road conditions. From the viewpoint of actual safety measures and expressway construction and management, the authors believe that the observation of brain activity in actual road experiments is essential.

As indicated in the introduction, it is a well-known fact that more accidents occur during deceleration than during acceleration, but the physiological reasons for this are not known. Our findings show that brain activation involved in voluntary eye movement control and in executive function is likely to increase while the driver is decelerating rapidly, especially at night. As these kinds of findings accumulate, it is possible that traffic safety measures can be developed that would help to increase or decrease brain activation during deceleration, by such means as redesign of deceleration lanes, or warning systems in places where sudden deceleration is likely to occur.

The correlations we found between prefrontal cortex activity and vehicle acceleration, do not necessarily imply a causal relationship. Time-course analysis will be required to clarify many issues in the relationship between the human brain and vehicle operation, including causality. 


\section{CONCLUSION}

This study demonstrated that prefrontal cortical activation increased with faster deceleration during actual road driving. This means that strong brain activation is required in situations when a driver has to brake rapidly. If the driver's prefrontal cortex does not work well during vehicle deceleration, the risk of accident may be increased. We also found that localized prefrontal cortical activation can be detected with good reproducibility by the simultaneous analysis of multiple hemodynamic indices with vector-based fNIRS, which makes it possible to detect both oxygen metabolic relationships and vascular relationships in the evaluation of brain activation.

\section{ACKNOWLEDGMENTS}

We thank Nagoya Electric Works Co., Ltd. who provided environmental maintenance for the experimental course and guard personnel to ensure safety. We would like to give special thanks to Patricia Yonemura for English editing.

\section{REFERENCES}

Baddeley, A., and Della Sala, S. (1996). Working memory and executive control. Philos. Trans. R. Soc. Lond. B Biol. Sci. 351, 1397-1403. doi: 10.1098/rstb.1996.0123

Fukushima, K., Sato, T., Fukushima, J., Shinmei, Y., and Kaneko, C. R. (2000). Activity of smooth pursuit-related neurons in the monkey periarcuate cortex during pursuit and passive whole-body rotation. J. Neurophysiol. 83, 563-587. Available online at: http://jn.physiology.org/content/83/1/563.full. pdf + html

Fukushima, K., Yamanobe, T., Shinmei, Y., Fukushima, J., Kurkin, S., and Peterson, B. W. (2002). Coding of smooth eye movements in three-dimensional space by frontal cortex. Nature 419, 157-162. doi: 10.1038/nature00953

Gamlin, P. D., and Yoon, K. (2000). An area for vergence eye movement in primate frontal cortex. Nature 407, 1003-1007. doi: 10.1038/35039506

Goldberg, M. E., Eggers, H. M., and Gouras, P. (1991). “The ocular motor system,” in Principles of Neural Science, 3rd Edn., eds E. R. Kandel, J. H. Schwartz, and T. M. Jessell (Norwalk, CT: Appleton \& Lange), 672-675.

Graydon, F. X., Young, R., Benton, M. D., Genik, 2nd. R. J., Posse, S., Hsieh, L., et al. (2004). Visual event detection during simulated driving: identifying the neural correlates with functional neuroimaging. Transport. Res. F7, 271-286. doi: 10.1016/j.trf.2004.09.006

Homae, F., Watanabe, H., Otobe, T., Nakano, T., Go, T., Konishi, Y., et al. (2010). Development of global cortical networks in early infancy. J. Neurosci. 30, 4877-4882. doi: 10.1523/JNEUROSCI.5618-09.2010

Kato, T. (2004). Principle and technique of NIRS imaging for human brain FORCE: fast-oxygen response in capillary event. Proc. ISBET 1270, 85-90. doi: 10.1016/j.ics.2004.05.052

Kato, T. (2006). Apparatus for Evaluating Biological Function. WO/2003/068070. Available online at: http://www.wipo.int/patentscope/search/en/WO20030 68070
Kato, T. (2007). Biofunctuon Diagnosis Device, Biofunction Diagnosis Method, Bioprobe, Bioprobe Wearing Tool, Bioprobe Support Tool, and Bioprobe Wearing Assisting Tool. WO/2006/009178. Available online at: http://www.wipo.int/ patentscope/search/en/WO2006009178

Kato, T., Yoshino, K., Oka, N., Yamamoto, K., and Takahashi, H. (2013). "First functional NIRS imaging of drivers' brain during driving in Japanese ShinTomei Expressway," in The 19th Annual Meeting of the Organization for Human Brain Mapping (Seattle, WA).

Kirilina, E., Jelzow, A., Heine, A., Niessing, M., Wabnitz, H., Bruhl, R., et al. (2012). The physiological origin of task-evoked systemic artefacts in functional near infrared spectroscopy. Neuroimage 61, 70-81. doi: 10.1016/j.neuroimage.2012.02.074

Pauling, L., and Coryell, C. D. (1936). The magnetic properties and structure of hemoglobin, oxyhemoglobin and carbonmonoxyhemoglobin. Proc. Natl. Acad. Sci. U.S.A. 22, 210-216. doi: 10.1073/pnas.22.4.210

Pierrot-Deseilligny, C., Milea, D., and Müri, R. M. (2004). Eye movement control by the cerebral cortex. Curr. Opin. Neurol. 17, 17-25. doi: 10.1097/01.wco.0000113942.12823.e0

Sano, M., Sano, S., Oka, N., Yoshino, K., and Kato, T. (2013). Increased oxygen load in the prefrontal cortex from mouth breathing: a vectorbased near-infrared spectroscopy study. Neuroreport, 24, 935-940. doi: 10.1097/WNR.0000000000000008

Takahashi, T., Takikawa, Y., Kawagoe, R., Shibuya, S., Iwano, T., and Kitazawa, S. (2011). Influence of skin blood flow on near-infrared spectroscopy signals measured on the forehead during a verbal fluency task. Neuroimage 57, 991-1002. doi: 10.1016/j.neuroimage.2011.05.012

Yamamoto, K., Takahashi, H., Kameoka, H., Tago, K., Okada, W., Tsuji, M., et al. (2012). "Verification of decline in the driver's concentration due to the control of light-emitting equipment," in 19th World Congress on ITS. No.AP-00346. (Vienna).

Yoshino, K., and Kato, T. (2012). Vector-based phase classification of initial dips during word listening using near-infrared spectroscopy. Neuroreport 23, 947-951. doi: 10.1097/WNR.0b013e328359833b

Yoshino, K., Oka, N., Yamamoto, K., Takahashi, H., and Kato, T. (2013). Functional brain imaging using near-infrared spectroscopy during actual driving on an expressway. Front. Hum. Neurosci. 7:882. doi: 10.3389/fnhum.2013.00882

Conflict of Interest Statement: The authors declare that the research was conducted in the absence of any commercial or financial relationships that could be construed as a potential conflict of interest.

Received: 06 October 2013; accepted: 09 December 2013; published online: 25 December 2013.

Citation: Yoshino K, Oka N, Yamamoto K, Takahashi H and Kato T (2013) Correlation of prefrontal cortical activation with changing vehicle speeds in actual driving: a vector-based functional near-infrared spectroscopy study. Front. Hum. Neurosci. 7:895. doi: 10.3389/fnhum.2013.00895

This article was submitted to the journal Frontiers in Human Neuroscience.

Copyright (c) 2013 Yoshino, Oka, Yamamoto, Takahashi and Kato. This is an openaccess article distributed under the terms of the Creative Commons Attribution License (CC BY). The use, distribution or reproduction in other forums is permitted, provided the original author(s) or licensor are credited and that the original publication in this journal is cited, in accordance with accepted academic practice. No use, distribution or reproduction is permitted which does not comply with these terms. 